\title{
Removal of craniopharyngioma via fronto-basal interhemispheric approach
}

\author{
HONGSHUN XING $^{1}$, HONGBO XING $^{2}$, PEIQUAN HUI $^{1}$ and BIN YANG ${ }^{3}$ \\ ${ }^{1}$ Department of Neurosurgery, Weifang People's Hospital, Weifang, Shandong 261041; ${ }^{2}$ Department of Stomatology, \\ Weihai Municipal Hospital, Weihai, Shandong 264200; ${ }^{3}$ Department of Neurosurgery, \\ Affiliated Hospital of Weifang Medical University, Weifang, Shandong 261031, P.R. China
}

Received February 18, 2016; Accepted May 11, 2016

DOI: $10.3892 / \mathrm{ol} .2016 .4555$

\begin{abstract}
The effectiveness and safety of fronto-basal interhemispheric approach in treating craniopharyngioma were evaluated. In this retrospective study, the safety and effectiveness of fronto-basal interhemispheric approach for surgical resection of craniopharyngioma in 20 patients was analyzed. Of the 20 patients, 12 were men and 8 were women, aged 15-65 years, with an average age of 42.5 years. The course of disease ranged from 1 to 36 months. The craniopharyngioma tumor was completely removed through surgical resection in 18 patients $(90 \%)$ and partially removed in 2 patients. The pituitary stalk was preserved in 18 patients and 1 patient succumbed during the study period due to large area pulmonary embolism. In conclusion, fronto-basal interhemispheric approach provides better access to the tumor that grows in the sella-hypothalamus and anterior third ventricle region. Using this approach, tumors can be resected while preserving the vital structures such as the pituitary stalk and hypothalamus.
\end{abstract}

\section{Introduction}

Craniopharyngioma is a common benign tumor of the sella region and it grows in the midline position of the sella-hypothalamus and is closely associated with the peripheral brain tissues, important nerves and blood vessel structures (1). It is difficult to achieve gross total removal of craniopharyngioma, thus, during the postoperative period, a considerable number of patients suffer from a high rate of mortality, tumor recurrence and postoperative complications (2). Selecting an appropriate surgical approach that allows preservation of vital structures including the hypothalamus is the key to achieving gross total removal and reduce postoperative complications $(3,4)$. It has

Correspondence to: Dr Bin Yang, Department of Neurosurgery, Affiliated Hospital of Weifang Medical University, 2428 Yuhe Road, Weifang, Shandong 261031, P.R. China

E-mail: yang_bin1212@163.com

Key words: craniopharyngioma, interhemispheric approach, gross total removal, pituitary stalk, post-operative complications been reported that, fronto-basal interhemispheric approach achieves a high rate of gross total removal of tumor and pituitary stalk preservation (5) and is being widely applied in clinical setting.

In the present study, the fronto-basal interhemispheric approach was employed in removing craniopharyngioma in 20 patients from January, 2012 to January, 2015.

\section{Patients and methods}

Patients. In this retrospective study, radiological images, operative studies and postoperative clinical follow-up data of 20 patients who underwent resection for craniopharyngioma via a fronto-basal interhemispheric approach between January, 2012 to January, 2015, were analyzed. Of the 20 patients, 12 were men and 8 women aged 15-65 years, with an average of 42.5 years. The course of disease ranged from 1 to 36 months. Comorbid conditions such as headache were present in 18 patients, 10 patients had visual acuity and visual field disorders, 2 patients had polydipsia and polyuria, 8 patients had nausea and vomiting, 5 patients had retardation and 4 patients had memory loss. All the patients underwent magnetic resonance imaging (MRI) or computed tomography (CT) scan prior to and following resection of craniopharyngioma.

Surgical technique. To perform resection using fronto-basal interhemispheric approach, the patient was positioned supine and the back was elevated $20^{\circ}$ to facilitate venous drainage. A coronal skin incision behind the hairline was carried out. Craniotomy was performed including the inner side of the frontal bone flap and lower end of arcus superciliaris and basis crania after making a single midline burrhole. The dura was cut open in an arc-shaped manner that allowed the dissection of basal interhemispheric fissure. This procedure exposed the end plate, optic chiasma and anterior communicating artery from the genus of corpus callosum. The tumor was exposed by opening of the end plate of the expanding hole. Subsequently, the craniopharyngioma tumor was removed under direct vision of the surgeon. The tumor that grew in the sella region was removed after abrading the olivary eminence via pneumatic drill and a large bone wax was used to seal the opened sphenoid sinus. During the 


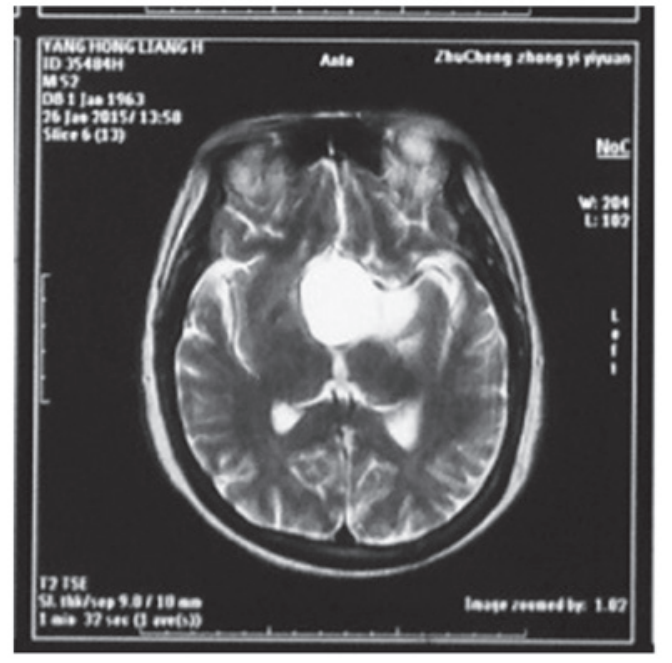

Figure 1. Brain magnetic resonance imaging (MRI) showing long T2 signal in the saddle area in a 45-year-old man hospitalized due to headache and vision disorder.

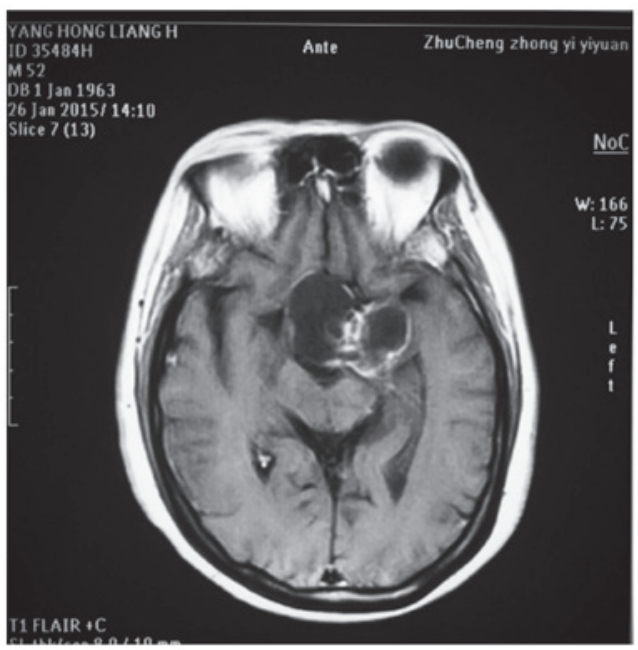

Figure 2. Head magnetic resonance imaging (MRI)-enhanced influence.

surgery, bipolar coagulation was used as little as possible to preserve the pituitary stalk.

\section{Results}

Pre-operative radiological diagnoses. The MRI and CT investigations identified tumors in the sellar-hypothalamus region in 10 patients, sellar-anterior third ventricle in 5 patients, interior sellar-sellar region in 2 patients and brainstem in 3 patients. The tumors were cystic and solid in structure in 13 patients, cystic in 4 patients and solid in 3 patients (Figs. 1 and 2).

Post-operative radiological diagnoses. The MRI and CT investigations of patients who underwent resection of craniopharyngioma revealed gross total removal of tumor was achieved in 18 cases (Fig. 3), tumor residue was present in 2 patients, and the pituitary stalk was preserved in 18 patients. Fig. 4 shows the incision of the fronto-basal interhemispheric approach. In the immediate post-operative period, vision was recovered or improved in 10 patients. The complications

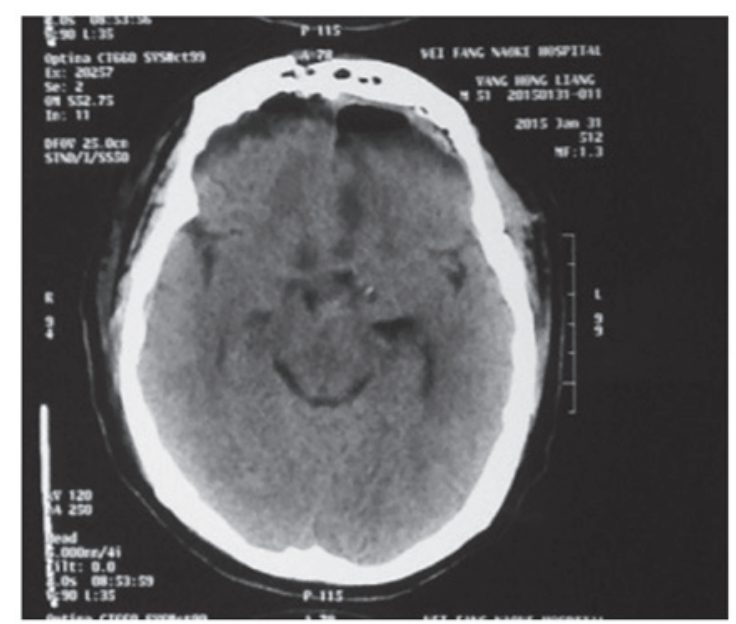

Figure 3. Postoperative radiological diagnosis shows gross total removal of the tumor.

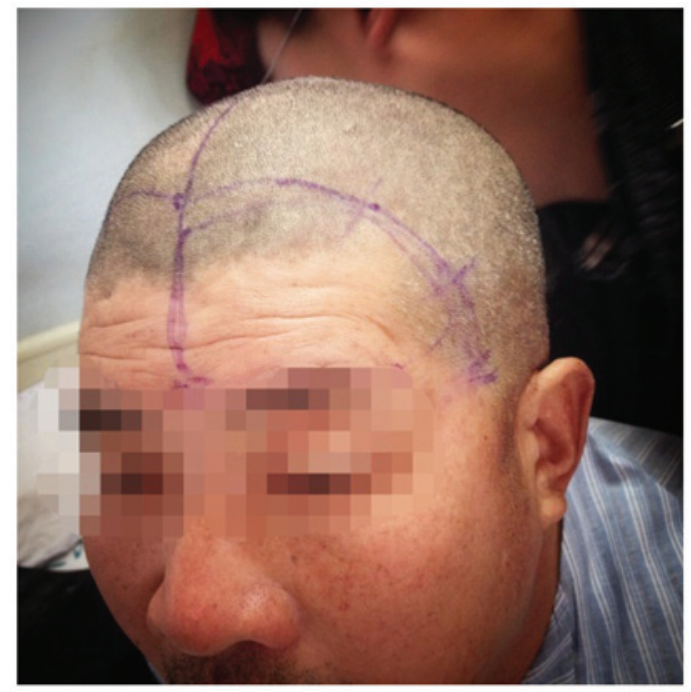

Figure 4. Incision of fronto-basal interhemispheric approach.

observed were polydipsia and polyuria in 15 patients and blood electrolyte disorder in 15 patients. A patient succumbed and the autopsy results confirmed that the patient due to large area pulmonary embolism.

\section{Discussion}

Craniopharyngioma is a common benign epithelial tumor and many studies have revealed that, patients can be cured if gross total removal of tumor is achieved. Nevertheless, craniopharyngioma is physically closely associated with hypothalamus and important blood vessels, which makes gross total removal difficult. Furthermore, partial removal is always accompanied with a higher recurrence rate in comparison with gross total removal (6).

Previously, craniopharyngioma was treated by surgery using the pterional and callosum interhemispheric approach. The pterional interhemispheric approach is time-consuming, and causes serious injury and profuse bleeding. Thus, its curative effects for tumors that invade sella, anterior third ventricle, 
brainstem and posterior circulation are poor. Furthermore during surgery, it inevitably involves the traction of internal carotid artery and optic nerves, which may lead to hemiplegia or even coma due to spasm of the internal carotid artery (7). The callosum interhemispheric approach may cause damage to the bilateral fornices in the anterior corpus callosum and when resection is carried out using this approach, it is difficult to discern the boundary of the tumor, sella region and optic chiasma. Thus, this approach leaves tumor residues, thereby achieving gross total removal of tumor is less effective. Hori et al (8) reported that the benefits of pterional and callosum interhemispheric approaches are limited in comparison with the fronto-basal interhemispheric approach.

Craniopharyngioma occurs in the pituitary stalk of the midline and grows along the midline. Since fronto-basal interhemispheric approach can reach the endplate and chiasmatic cistern (9) directly, it is a convenient way to approach the tumor. In the present study, during surgical resection, no significant structural damage occurred.

The fronto-basal interhemispheric approach was effective in removing the tumors that grow from the sellar region to the anterior third ventricle and even for tumors that extended into the third ventricle. In the present study, two patients had a tumor in their anterior third ventricle and were successfully resected and the operative field was good. Even a large tumor that pressed the interpeduncular cistern and grew into the anterior pontine cistern of dorsum sella was gross totally removed under direct vision of the surgeon.

In the present study, one patient who had tumor closely attached to the top and branches of basal artery and showed bulk-shaped calcification had gross total removal and no recurrence occurred after surgery. For the tumors that grow towards the interior sella, the fronto-basal interhemispheric approach provides a relatively large visual angle from below and anterior direction. If the tumors grow towards the sphenoid sinus via expanded sella turcica, it results in a visual blind area in the anterior wall of sella turcica. A pneumatic drill can be used to abrade the tuberculum sellae to remove the tumor inside the sellar region. In the current study, three patients had tumors growing towards sphenoid sinus and the tuberculum sellae was abraded to achieve tumor removal, but two patients showed tumor residues after surgery. The results showed that, for the large craniopharyngioma in the midline area of the sella region, double frontal craniotomy combined with the fronto-basal interhemispheric approach provides more surgical space. Sometimes, the tumor protrudes towards internal carotid or spreads across internal carotid artery. In such situations, use of subfrontal approach creates a larger visual field for the surgeon.

Craniopharyngioma originates from the pituitary stalk and tumor residues in this area are always the source of tumor recurrence. The gross total removal of the tumors, while preserving the pituitary stalk has been a burden to surgeons for a long time (10). The fronto-basal interhemispheric approach is useful for the removal of tumor tissues in the pituitary stalk from below and posterior area or anterior area of chiasma opticum. The retention rate of the pituitary stalk of pterion approach was approximately $33 \%$ (11). However, in the present study using the fronto-basal interhemispheric approach, the retention rate of the pituitary stalk reached $90 \%$ (18 patients) and most patients experienced diabetes insipidus after surgery in a transient manner.

In comparison with the pterion or callosum approaches, the fronto-basal interhemispheric approach requires experienced surgeons. This approach involves stretching of cerebral tissues, thus damage arising from stretching the hypothalamus were mild but for the frontal lobe were severe. In the immediate post-operative period, the patients experienced mental problems. During all the surgeries, the surgeon was careful in protecting the fine perforating arteries that originate from the anterior communicating artery and supplying blood to the hypothalamus and basal ganglia.

In conclusion, the fronto-basal interhemispheric approach is safer and provides more direct surgical vision that is benefiical in identifying the pituitary stalk and reducing damage to the olfactory nerve, optic nerve, internal carotid artery, hypothalamus and other important structures. The immediate post-operative complications are also fewer and this favors rapid recovery of patients.

\section{References}

1. Liu JK, Christiano LD, Gupta G and Carmel PW: Surgical nuances for removal of retrochiasmatic craniopharyngiomas via the transbasal subfrontal translamina terminalis approach. Neurosurg Focus 28: E6, 2010.

2. Kassam AB, Prevedello DM, Thomas A, Gardner P, Mintz A, Snyderman $C$ and Carrau R: Endoscopic endonasal pituitary transposition for a transdorsum sellae approach to the interpeduncular cistern. Neurosurgery 62: 57-72, 2008.

3. Pettorini BL, Frassanito P, Caldarelli M, Tamburrini G, Massimi L and Di Rocco C: Molecular pathogenesis of craniopharyngioma: switching from a surgical approach to a biological one Neurosurg Focus 28: E1, 2010.

4. Campbell PG, McGettigan B, Luginbuhl A, Yadla S, Rosen M and Evans JJ: Endocrinological and ophthalmological consequences of an initial endonasal endoscopic approach for resection of craniopharyngiomas. Neurosurg Focus 28: E8, 2010.

5. Kassam AB, Gardner PA, Snyderman CH, Carrau RL, Mintz AH and Prevedello DM: Expanded endonasal approach, a fully endoscopic transnasal approach for the resection of midline suprasellar craniopharyngiomas: a new classification based on the infundibulum. J Neurosurg 108: 715-728, 2008.

6. Nishimoto A, Matsuhisa T, Kunishio K, Maeshiro T, Furuta $\mathrm{T}$ and Ohmoto T: Craniopharyngioma: Early and long term recurrence after partial removal. J Neurol Neurosurg Psychiatry 58: 111-112, 1995 .

7. Kunihiro N, Goto T, Ishibashi K and Ohata K: Surgical outcomes of the minimum anterior and posterior combined transpetrosal approach for resection of retrochiasmatic craniopharyngiomas with complicated conditions. J Neurosurg 120: 1-11, 2014.

8. Hori T, Kawamata T, Amano K, Aihara Y, Ono M and Miki N: Anterior interhemispheric approach for 100 tumors in and around the anterior third ventricle. Neurosurgery 66: 65-74, 2010.

9. Jung TY, Jung S, Choi JE, Moon KS, Kim IY and Kang SS: Adult craniopharyngiomas: surgical results with a special focus on endocrinological outcomes and recurrence according to pituitary stalk preservation. J Neurosurg 111: 572-527, 2009.

10. Garrè ML and Cama A: Craniopharyngioma: modern concepts in pathogenesis and treatment. Curr Opin Pediatr 19: 471-479, 2007.

11. Shi XE, Wu B, Zhou ZQ, Fan T and Zhang YL: Microsurgical treatment of craniopharyngiomas: report of 284 patients. Chin Med J (Engl) 119: 1653-1663, 2006. 